\title{
Assembly of AUF1 with elF4G-poly(A) binding protein complex suggests a translation function in AU-rich mRNA decay
}

\author{
JIN-YU LU, ${ }^{1}$ NAOMI BERGMAN, NAVID SADRI, and ROBERT J. SCHNEIDER \\ Department of Microbiology, New York University School of Medicine, New York, New York 10016, USA
}

\begin{abstract}
An AU-rich element (ARE) located in the 3 '-untranslated region of many short-lived mRNAs functions as an instability determinant for these transcripts. AUF1/hnRNP D, an ARE-binding protein family consisting of four isoforms, promotes rapid decay of ARE-mRNAs. The mechanism by which AUF1 promotes rapid decay of ARE-mRNA is unclear. AUF1 has been shown to form an RNase-resistant complex in cells with the cap-initiation complex and heat shock proteins $\mathrm{Hsp70}$ and $\mathrm{Hsc70}$, as well as other unidentified factors. To understand the function of the AUF1 complex, we have biochemically investigated the association of AUF1 with the components of the translation initiation complex. We used purified recombinant proteins and a synthetic ARE RNA oligonucleotide to determine the hierarchy of protein interactions in vitro and the effect of AUF1 binding to the ARE on the formation of protein complexes. We demonstrate that all four AUF1 protein isoforms bind directly and strongly to initiation factor eIF4G at a C-terminal site regardless of AUF1 interaction with the ARE. AUF1 is shown to directly interact with poly(A) binding protein (PABP), both independently of eIF4G and in a complex with eIF4G. AUF1-PABP interaction is opposed by AUF1 binding to the ARE or Hsp70 heat shock protein. In vivo, AUF1 interaction with PABP does not alter PABP stability. Based on these and other data, we propose a model for the molecular interactions of AUF1 that involves translation-dependent displacement of AUF1-PABP complexes from ARE-mRNAs with possible unmasking of the poly(A) tail.
\end{abstract}

Keywords: AUF1; hnRNP D; mRNA decay; poly(A) binding protein (PABP); elF4G; AU-rich element (ARE)

\section{INTRODUCTION}

ARE sequences located in the $3^{\prime}$ untranslated region (3'-UTR) of short-lived higher eukaryotic mRNAs are responsible for their rapid turnover. AREs generally consist of repeating pentamers of the sequence AUUUA, which promotes the rapid cytoplasmic degradation of cytokine and proto-oncogene mRNAs (Shaw and Kamen 1986; Stoecklin et al. 2000; Guhaniyogi and Brewer 2001). Accelerated decay of ARE-mRNAs is thought to proceed by rapid deadenylation, which, in turn, leads to rapid degradation of the body of the mRNA (Brewer and Ross 1988; Wilson and Treisman 1988; Shyu et al. 1989, 1991;

\footnotetext{
${ }^{1}$ Present address: BCMM 231, Howard Hughes Medical Institute, Yale University School of Medicine, New Haven, CT 06536, USA.

Reprint requests to: Robert J. Schneider, Department of Microbiology, New York University School of Medicine, 550 First Avenue, New York, NY 10016, USA; e-mail: robert.schneider@med.nyu.edu; fax: (212) 263-8276. Article published online ahead of print. Article and publication date are at http://www.rnajournal.org/cgi/doi/10.1261/rna.2308106.
}

Xu et al. 1997; Ford and Wilusz 1999). The ARE is often sufficient to confer rapid cytoplasmic degradation of an mRNA, and its removal often stabilizes short-lived mRNAs (Guhaniyogi and Brewer 2001). In many but not all systems, active translation of the ARE-mRNA itself stimulates its degradation (Savant-Bhonsale and Cleveland 1992; Aharon and Schneider 1993; Curatola et al. 1995; Winstall et al. 1995), although there is little mechanistic understanding of this event. Equally puzzling is the poorly understood connection between ubiquitination-proteasome AUF1 activity, and the rapid degradation of ARE-mRNAs (Pouch et al. 1995; Schmid et al. 1995; Petit et al. 1997; Laroia et al. 1999, 2002).

The AUF1 isoforms each interact with some specificity for the different types of ARE sequences, which has been attributed to exon insertions that likely alter AUF1 conformation (Wagner et al. 1998; Laroia and Schneider 2002). Different AUF1 family members have been reported to have multiple functions, including transcriptional activation (Brys and Maizels 1994; Fuentes-Panana et al. 2000), 
interaction with nucleolin (Hanakahi et al. 1997), telomeric single-stranded DNA (Eversole and Maizels 2000), and components of the translation initiation apparatus (Laroia et al. 1999). All four AUF1 isoforms also associate with hnRNP A1 (Pinol-Roma et al. 1988), which is involved in mRNA transport (Pinol-Roma and Dreyfuss 1992) and splicing (Mayeda and Krainer 1992).

The ability of the ARE to regulate mRNA stability has been shown to involve interaction with several different AU-rich RNA-binding proteins. At least three ARE-binding proteins have been found to regulate the rapid degradation of ARE-mRNAs. Members of a family of HU-binding proteins, particularly $\mathrm{HuR}$ and $\mathrm{HuB}$, bind to many different AU-rich sequences (Ma et al. 1996) and stabilize AREcontaining reporter mRNAs (Fan and Steitz 1998; Peng et al. 1998). Knockdown of endogenous HuR expression by antisense RNA decreases half-lives of some ARE-containing mRNAs (Levy et al. 1998; Rodriguez-Pascual et al. 2000; Wang et al. 2000), consistent with a role in stabilization of ARE-mRNAs. The protein tristetraprolin (TTP) (Carballo et al. 2000) promotes destabilization of TNF and GM-CSF mRNAs, as determined in knockout mice (Taylor et al. 1996; Carballo et al. 2000), and in tissue culture by ectopic overexpression of TTP protein (Lai et al. 2000). The AUrich RNA-binding protein known as KSRP has been implicated in ARE-mRNA decay by virtue of its interaction with the exosome, a large multisubunit particle that mediates degradation of the mRNA body but not deadenylation (Chen et al. 2001).

The ARE/poly(U)-binding/degradation factor, known as AUF1 (Brewer 1991) or hnRNP D (Wagner et al. 1998), also plays a role in ARE-mRNA decay. AUF1 consists of four isoforms produced by alternate splicing of a single mRNA to produce a $37-\mathrm{kDa}$ core protein (p37), a $40-\mathrm{kDa}$ protein (p40) with an N-terminal 19-amino-acid insertion of exon 2, a 42-kDa protein (p42) with a C-terminal 49amino-acid insertion of exon 7 , and a $45-\mathrm{kDa}$ protein (p45) containing both exon 2 and 7 insertions (for review, see Guhaniyogi and Brewer 2001). The four AUF1 isoforms bind to different ARE sequences with different affinities, attributable to the presence of the exon insertions (Kajita et al. 1995; Wagner et al. 1998). In addition, purified p37 and p40 AUF1 proteins enhance c-myc mRNA turnover in an in vitro decay system (Brewer 1991), and the p37 isoform displays the highest destabilizing activity for reporter AREmRNA (Loflin et al. 1999; Sarkar et al. 2003b). There is little understanding of the interactions among AUF1 family members with each other and with other proteins that function in mRNA decay. p37 AUF1 has been shown to assemble as oligomers on AU-rich RNA (Wilson et al. 1999). The interaction among isoforms has not been studied. One group has found the p37 and p40 isoforms in association with the mammalian exosome (Chen et al. 2001). AUF1 has also been reported to be a component of the $\alpha$-globin mRNA stabilization complex (Kiledjian et al.
1997). More recently, AUF1 was shown to bind lactate dehydrogenase (LDH) and Hsp70 on polysomal mRNAs containing an ARE, to which LDH can also bind (Pioli et al. 2002). We previously demonstrated that all four AUF1 isoforms interact with the cap-initiation complex group of proteins that contain translation initiation factors eIF4G, eIF4E, eIF4A, and PABP, as well as $\mathrm{Hsp} / \mathrm{Hsc}-70$ proteins (Laroia et al. 1999).

We are interested in the interaction of AUF1 isoforms with proteins that might be involved in regulating AREmRNA decay, particularly those of the translation apparatus. An understanding of these interactions could provide a link between the rapid turnover of ARE-mRNAs and the requirement (though not absolute) for mRNA translation and the ubiquitin-proteasome system (Sarkar et al. 2003b). Here we have investigated the direct interactions between the four AUF1 isoforms and members of the cap-initiation complex in vitro. To facilitate characterization and mapping analysis, we used recombinant proteins and a synthetic ARE to determine the hierarchy of protein interactions, and the effect of AUF1 binding on the ARE on protein-protein interactions. We demonstrate that all four AUF1 protein isoforms can interact directly and strongly with eIF4G, binding at a C-terminal site on eIF4G that is not known to be occupied by other associated proteins. AUF1-eIF4G interaction is not altered by AUF1 interaction with the ARE. AUF1 is also shown to directly interact with PABP on or off eIF4G. The PABP-AUF1 interaction is reduced by AUF1 binding to the ARE or by the presence of Hsp70 heat shock protein, and without influencing PABP stability in vivo. Based on these and other data, we present a model for the molecular interactions of AUF1 that impact on translational regulation of ARE-mRNA decay.

\section{RESULTS}

\section{The p37 AUF1 isoform interacts directly and strongly in vitro with the $C$ terminus of eIF4G}

Studies have demonstrated that p37 AUF1 exists as monomers and dimers in solution but forms tetramers and higher-molecular-weight complexes when bound to an ARE on mRNA (DeMaria et al. 1997; Wilson et al. 1999). Thus, the ARE likely initiates the assembly of AUF1 highermolecular-weight complexes. In this report, we investigated the interaction of ARE-bound and free AUF1 with components of the cap-initiation complex. Recombinant p37 AUF1 was expressed in Escherichia coli and purified (see Materials and Methods for details). The ability of AUF1 to assemble in vitro into ARE-protein complexes, which were used in this study, was demonstrated by incubation of p37 AUF1 with a synthesized RNA corresponding to the TNF ARE, which was resolved by gel electrophoresis and detected by immunoblot analysis of AUF1 as described (Wilson et al. 1999). Reactions were treated with the 
chemical cross-linking agent DSP prior to electrophoresis to covalently stabilize complexes (Wilson et al. 1999). In the absence of the ARE, abundant 37-kDa AUF1 monomers were observed, as well as some $70-\mathrm{kDa}$ dimers, but no higher-molecular-weight AUF1 complexes could be detected (Fig. 1). Addition of $5 \mu \mathrm{M}$ ARE RNA oligonucleotide to the reaction strongly promoted formation of dimers and trimers of AUF1, which was not further enhanced at $10 \mu \mathrm{M}$ ARE, as shown previously (Wilson et al. 1999). At $1 \mu \mathrm{M}$ ARE, $\sim 50 \%$ of the maximum level of AUF1-ARE complex formation $\left(\mathrm{C}_{50}\right)$ was observed. We therefore used the $1 \mathrm{mM}$ concentration for all subsequent studies except where noted. It is important to note that AUF1 complexes were not observed with addition of poly $(G)$, even with chemical cross-linking (data not shown). Cross-linking permitted electrophoretic resolution, and complexes were not cross-linked in other experiments. Cross-linking also reduces the immunoreactivity of AUF1 complexes (G. Wilson, pers. comm.). Hence, while addition of the ARE in subsequent experiments results in the formation of ARE-AUF1 complexes, the extent as shown here is an underestimate.

Given the ability of recombinant p37 AUF1 protein to interact with the ARE in vitro and with components of the cap-initiation complex in vivo (Laroia et al. 1999), and its ability to accelerate ARE-mRNA decay, we investigated the direct protein-binding partners of AUF1 in the cap complex. Previous studies showed that immunoprecipitation of RNA-free AUF1 complexes resulted in coisolation of eIF4G, PABP, and Hsp-Hsc-70 proteins (Laroia et al. 1999). Therefore, one or more of these proteins is likely a direct interaction partner. We first probed the potential interaction between p37 AUF1 and initiation factor eIF4G, since it is a large molecular scaffold for assembly of cap complexes. It has not been possible to produce recombinant, undegraded, full-length eIF4G in sufficient quantities for in vitro binding studies, but the protein can be produced and

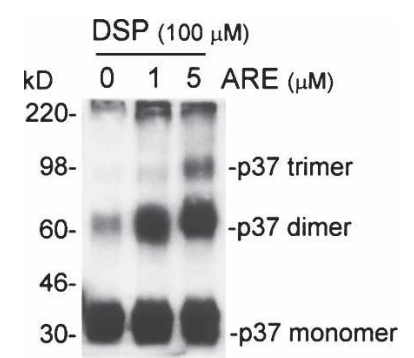

FIGURE 1. Oligomerization of p37 AUF1 promoted by ARE RNA. Purified p37 AUF1 protein $(1 \mu \mathrm{M})$ was incubated with or without an RNA oligonucleotide corresponding to the TNF ARE at concentrations shown and subjected to protein-protein cross-linking with DSP, and complexes were resolved by nonreducing PAGE. AUF1 protein complexes were detected by immunoblot analysis using an antibody that recognizes all four isoforms. Following cross-linking, the AUF1 proteins were not well recognized by the AUF1 polyclonal antibody. The size and deduced higher-order interaction of p37 AUF1 proteins were based on molecular weight standards. Data were quantified by densitometry. purified as three GST-tagged fragments corresponding to $\mathrm{N}$-terminal (N4G), middle (M4G), and C-terminal (C4G) thirds of the protein (Cuesta et al. 2000a,b). In vitro interaction analyses were conducted with equimolar amounts of purified His-tagged p37 AUF1 protein and fragments of GST-eIF4G in the presence of $0.5 \%$ NP-40 detergent and $100 \mu \mathrm{g} / \mathrm{mL}$ bovine serum albumin (BSA) to select for stringent protein-protein interactions (Fig. 2). p37 AUF1 strongly interacted with the C-terminal (C4G) fragment of eIF4G, but not with the $\mathrm{N}$-terminal or middle fragments. There was no interaction between GST itself in the absence of the C4G eIF4G fragment and p37 AUF1. There are five isoforms of eIF4G, representing progressive in-frame N-terminal extensions (Bradley et al. 2002; Byrd et al. 2002). Although the major expressed form in vivo and the N4G fragment used here do not represent full-length eIF4G, we also tested an extended N-terminal eIF4G fragment (extN4G) for interaction with p37 AUF1 (Fig. 2). The $\mathrm{N}$-terminal extended form of eIF4G did not interact with AUF1. Thus, p37 AUF1 interacts directly and strongly with the $\mathrm{C}$ terminus of eIF4G.

To determine whether AUF1 formation of complexes on the ARE influences interaction with eIF4G, $1 \mu \mathrm{M}$ of a synthesized copy of the 38-nt TNF $\alpha$ ARE was added to reactions containing His-p37-AUF1 and GST-eIF4G fragments to drive complex formation (Fig. 2A). Addition of the ARE promoted multimer formation (Fig. 2B) but did not detectably decrease p37 AUF1 interaction with the C terminus of eIF4G (shown in Fig. 2A). As the complexes were recovered by p37 affinity chromatography, these results suggest that AUF1 can interact with both eIF4G and the ARE in vitro. AUF1 might therefore promote crosstalk between the signals in the $3^{\prime}$-UTR that promote mRNA decay and the translational machinery.

The ability of the other three isoforms of AUF1 to interact with the C-terminal fragment of eIF4G was also examined. Each AUF1 isoform was produced as a T7/His 6 protein in E. coli and purified as recombinant proteins. Equal amounts of each AUF1 isoform were added to an in vitro interaction assay with equal molar amounts of the GST-C4G eIF4G protein fragment. GST was recovered by glutathione-Sepharose chromatography, and associated proteins were identified by SDS-PAGE and immunoblot analysis (anti-T7 for AUF1 protein isoforms, anti-GST for C4G) (Fig. 3). The p37 AUF1 isoform interacted most strongly with the C terminus of eIF4G, followed by the $\mathrm{p} 40$ isoform $(\sim 30 \%$ that of $\mathrm{p} 37)$. There was a weaker interaction detected between the $\mathrm{p} 45(\sim 15 \%)$ and $\mathrm{p} 42$ $(\sim 20 \%)$ AUF1 isoforms and the C terminus of eIF4G, despite the nearly equivalent level of input AUF1 protein isoforms (Fig. 3). There was no interaction between $\mathrm{C} 4 \mathrm{G}$ and GST itself. Interestingly, ARE-mRNA decay is promoted most strongly by the p37 AUF1 isoform followed by $\mathrm{p} 40$, and overexpression of $\mathrm{p} 37$ AUF1 relieves the translation requirement for activation of ARE-mRNA decay 

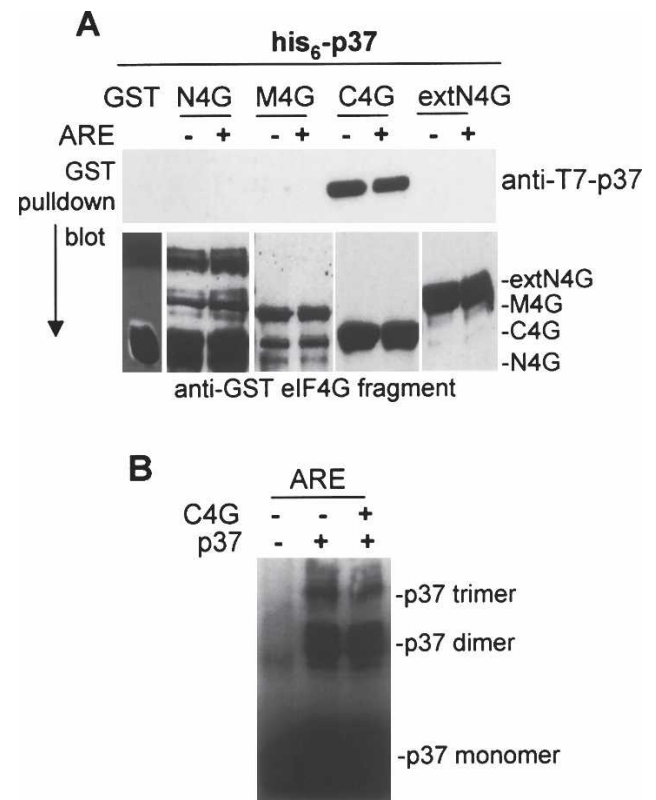

FIGURE 2. p37 AUF1 directly binds to the C terminus of eIF4G independently of ARE interaction. (A) Reactions contained $1 \mu \mathrm{g}$ of purified GST proteins or GST-eIF4G protein fragments corresponding to the N-terminal third (N4G), the middle third (M4G), the Cterminal third (C4G), or the full-length N-terminal eIF4G third (extN4G) of eIF4G, and $0.25 \mu \mathrm{M}$ his $_{6}$-tagged p37 AUF1 protein. After $1 \mathrm{~h}$, glutathione-Sepharose $4 \mathrm{~B}$ resin was added for $1 \mathrm{~h}$ to recover GST proteins. After extensive washing, proteins that remained bound to the beads were analyzed by SDS-PAGE followed by immunoblot analysis and autoradiography. One micromolar synthetic TNF $\alpha$ ARE RNA oligonucleotide was added to reactions as indicated. (B) Reactions were carried out as described above using GST-C4G, ARE, and p37 AUF1. Complexes were cross-linked and analyzed as described for Figure 1. Typical results of at least three independent experiments are shown.

(Sarkar et al. 2003b). The possible relevance of these independent observations is discussed below. Given that the strongest interaction with eIF4G was with the p37 AUF1 isoform, and that p37 is also most closely associated with ARE-mRNA decay, our studies focused exclusively on the in vitro interactions of this isoform with members of the cap-initiation complex and the ARE.

\section{Identification of p37 AUF1-binding domain for the $C$ terminus of elF4G}

Several structural elements of AUF1 have been identified. AUF1 proteins contain two nonidentical RNA-binding domains (RBDs), and the $\mathrm{N}$ and $\mathrm{C}$ termini of AUF1 are thought to mediate protein-protein interactions and nucleocytoplasmic shuttling activities (Wilson et al. 2001; Sarkar et al. 2003a). To identify the domain of the interaction site of p37 AUF1 with the C terminus of eIF4G, three truncation mutants of AUF1 were constructed as His-fusion proteins (Fig. 4A). AUF1 mutants were developed corresponding to deletion of the $\mathrm{N}$-terminal 78 amino acids

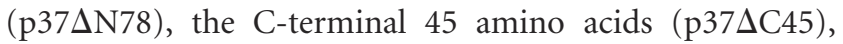
and both the $\mathrm{N}$ - and C-terminal regions (p37 $\Delta \mathrm{NC}$ ), leaving the two RBDs unaffected. Truncation mutants were expressed in E. coli, purified, and used for in vitro interaction analysis with the GST-C4G fragment of eIF4G. GST-eIF4G fragments were recovered by glutathione-Sepharose chromatography and detected by immunoblot analysis of associated proteins (Fig. 4B). Wild-type p37 AUF1 interacted strongly with C4G as shown above. The p37 3 N78 mutant bound to GST-C4G with an apparently reduced affinity, and there was no detectable interaction with the p37 $\triangle \mathrm{C} 45$ or p37 $\mathrm{NC}$ mutants. GST-PABP (purified and RNA-free) also bound strongly to full-length p37 AUF1 and apparently far more than the p $37 \Delta$ N78 mutant protein (Fig. 4B).

The apparently reduced levels of p37 $\Delta$ N78 recovered from GST-C4G and GST-PABP pull-down were found to result from poor antibody detection of the $\mathrm{N}$-terminal AUF1 truncation mutant, not from decreased binding to GST-C4G. Large N-terminal truncations of AUF1 are much less efficiently recognized by anti-AUF1 antibodies compared to the full-length p37 AUF1 protein. This was demonstrated by Coomassie blue staining and immunoblot analysis of the same amount of full-length or truncated AUF1 proteins (Fig. 4C). Thus, the C-terminal 45-amino acid domain of AUF1 is essential for interaction with eIF4G and PABP.

\section{p37 AUF1 and PABP directly interact in vitro in the absence of an ARE}

Several studies suggested that the ARE promotes a more rapid rate of mRNA deadenylation (Shyu et al. 1989, 1991; Schiavi et al. 1994; Xu et al. 1997), which has been linked to accelerated mRNA turnover. PABP is thought to impair

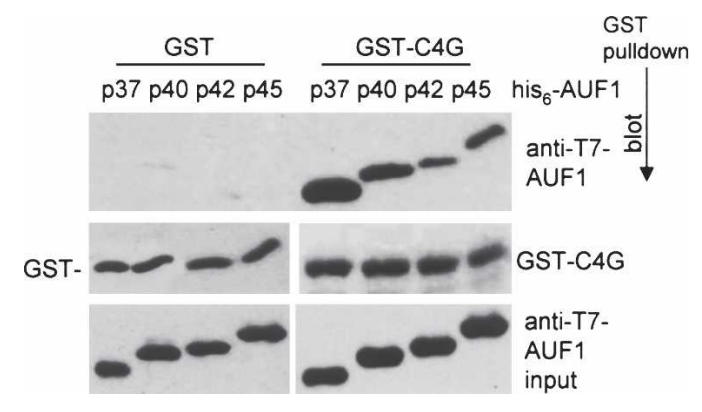

FIGURE 3. The p37 AUF1 isoform demonstrates the highest-affinity binding to the C-terminal fragment of eIF4G. One microgram of GST or GST-C4G fusion proteins was incubated with the four different $\mathrm{His}_{6}$-tagged AUF1 isoforms as indicated $(0.15 \mu \mathrm{g}$ of protein) without the ARE RNA oligonucleotide at $4^{\circ} \mathrm{C}$ for $1 \mathrm{~h}$. GST-fusion protein was recovered, and bound AUF1 proteins were detected by immunoblot analysis. Input his ${ }_{6}$-AUF1 proteins are shown. GST-C4G recovered by glutathione beads is shown, detected by immunoblot analysis using antibodies to GST. Autoradiograms were quantified by densitometry. 
A

\begin{tabular}{|c|c|c|c|}
\hline & RBD1 & RBD2 & $Q$ \\
\hline p37AUF1 & I & & 1 \\
\hline p37 $\triangle N 78$ & ᄃ & & I \\
\hline p37 $\triangle \mathrm{C} 45$ & I & & \\
\hline p37 $\triangle N C$ & & & \\
\hline
\end{tabular}

B

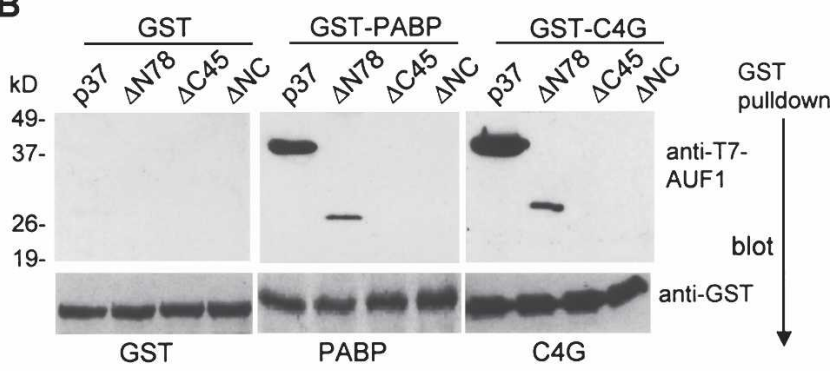

C

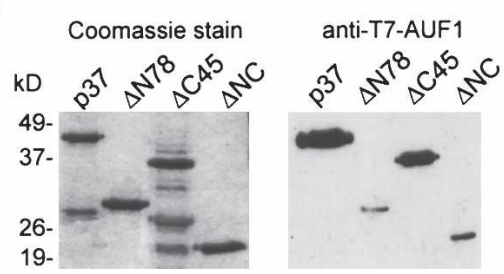

FIGURE 4. Mapping interaction domain of AUF1 with PABP and the C-terminal part of eIF4G. (A) Schematic representation of structures of various p37 AUF1 deletion mutant proteins. (RBD) RNA-binding domains; (Q) glutamine-rich region. (B) GST, GST$\mathrm{C} 4 \mathrm{G}$, and GST-PABP proteins were incubated with purified his ${ }_{6}-\mathrm{p} 37$ AUF1 deletion mutant proteins. After extensive washing, bound his $_{6}$ tagged proteins were detected by anti-T7 antibody. The recovered GST-proteins are shown, detected by immunoblot of GST. (C) Input his $_{6}$-tagged AUF1 proteins were also detected by Coomassie blue staining of the SDS-PAGE gel and by Western immunoblot analysis using anti-T7 antibody. Typical results of at least three independent experiments are shown and quantified by densitometry.

mRNA decay by protecting the poly(A) tail from deadenylation, a first step in mRNA turnover (Guhaniyogi and Brewer 2001). In addition, p37 AUF1, and to a lesser extent p40 isoforms, have been shown to undergo ubiquitination and rapid turnover by proteasome mediated degradation, which might be associated with their ability to promote rapid decay of ARE-mRNAs (Laroia et al. 1999, 2002). AUF1 complexes in vivo have also been shown to include $\mathrm{PABP}$ and $\mathrm{Hsp} / \mathrm{Hsc70}$, which can participate in proteasome presentation of proteins. Studies were therefore carried out to determine whether p37 AUF1 interacts directly in vitro with $\mathrm{PABP}$, which could be involved in more rapid deadenylation of ARE-mRNAs.

GST-p37 AUF1 and His-PABP were expressed in E. coli and used at equimolar amounts for in vitro interaction analysis, then recovered by glutathione-Sepharose binding of GST-p37 AUF1 proteins and interactants detected by immunoblot analysis (Fig. 5A). The TNF $\alpha$ ARE RNA oligonucleotide was added at a concentration of $1 \mu \mathrm{M}$. Since Hsp70 uses ATP hydrolysis for chaperone activity (Bukau and Horwich 1998), and Hsp70 is a component of the AUF1 complex formed in vivo, both ATP ( $2 \mathrm{mM})$ and Hsp70 protein were added to some reactions. There was no interaction between PABP and GST alone (Fig. 5A, cf. lanes 1 and 2). Notably, there was a strong direct interaction between PABP and p37 AUF1, which was significantly decreased (approximately fivefold) by addition of the ARE (Fig. 5A, cf. lanes 3 and 4). ATP addition slightly increased PABP interaction with p37 AUF1 (approximately twofold) (Fig. 5A, cf. lanes 3-5), which is likely caused by copurification of very small amounts of E. coli GroEL chaperone protein, which can stimulate native refolding of purified proteins, but this was also significantly decreased by addition of the ARE. Moreover, addition of ATP did not alter Hsp70 interaction with AUF1 (Fig. 5B), again suggesting that ATP and Hsp70 are not simply promoting native folding of purified proteins in this system. As shown in Figure 1, control studies demonstrated that addition of the ARE RNA enhanced formation of AUF1-ARE complexes and dimerization of AUF1. Importantly, addition of Hsp70 protein decreased the interaction between PABP and AUF1 (approximately two- to threefold) (Fig. 5A, cf. lanes 6-9). However, the interaction PABP-AUF1-Hsp70 could be recovered by the absence of the ARE RNA (Fig. 5A, cf. lanes 5-10). The presence of Hsp70 also moderately reduced the interaction of p37 AUF1 with PABP, even

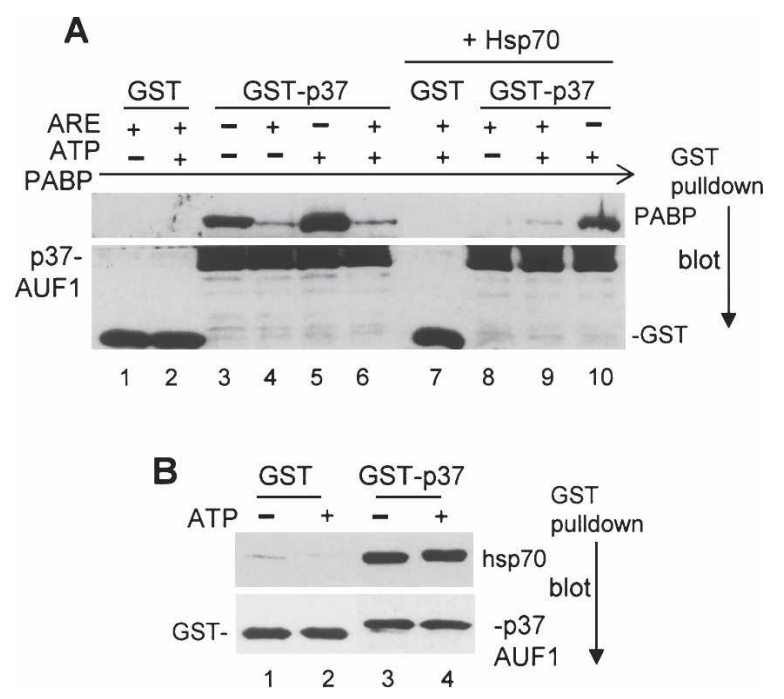

FIGURE 5. Direct interaction of $\mathrm{p} 37$ AUF1 with PABP is regulated by binding to the ARE and Hsp70. (A) $0.5 \mu \mathrm{g}$ of his ${ }_{6}$-tagged PABP was incubated with GST-p37 protein in a binding buffer for $1 \mathrm{~h}$, and $1 \mu \mathrm{M}$ TNF $\alpha$ ARE RNA oligonucleotide, $2 \mathrm{mM}$ ATP, and $0.5 \mu \mathrm{g}$ of recombinant Hsp70 were added as indicated. After GST recovery using GSH-glutathione beads, bound PABP was detected by antiPABP antibody (10E10). Recovery of GST-fusion proteins was carried out using anti-GST antibody. (B) Reactions contained GST-p37, Hsp70, and ATP as described above. GST-p37 was recovered by glutathione beads, and the interaction dependence of Hsp70 with AUF1 was determined by immunoblot analysis. Autoradiograms were quantified by densitometry. Typical results of at least three independent experiments are shown. 
without the addition of the ARE RNA (two- to threefold) (Fig. 5A, lanes 5,10). These results therefore indicate that AUF1 directly binds PABP, but this interaction is disrupted by simultaneous interaction of AUF1 with the ARE. It should be noted that extensive treatment of PABP with a mixture of RNases did not alter its interaction with AUF1 (data not shown). We can therefore conclude that AUF1 cannot simultaneously interact with both the ARE and $\mathrm{PABP}$, at least in this experimental system.

To determine whether the presence of a poly(A) tail influences interaction of PABP with AUF1, homopolymeric poly(A) RNA of $\sim 500$ residues was added to reactions described above at a ratio determined to be sufficiently large to bind most of the PABP present in reactions (data not shown). Addition of the ARE decreased PABP-AUF1 interaction by approximately fivefold, determined as described above (Fig. 6A). Addition of poly(A) did not have any influence on AUF1-PABP interaction in the absence of the ARE (Fig. 6A, cf. lanes 1 and 3). However, PABP interaction with AUF1 was decreased by the presence of the ARE, and strongly so with addition of poly(A). These

A
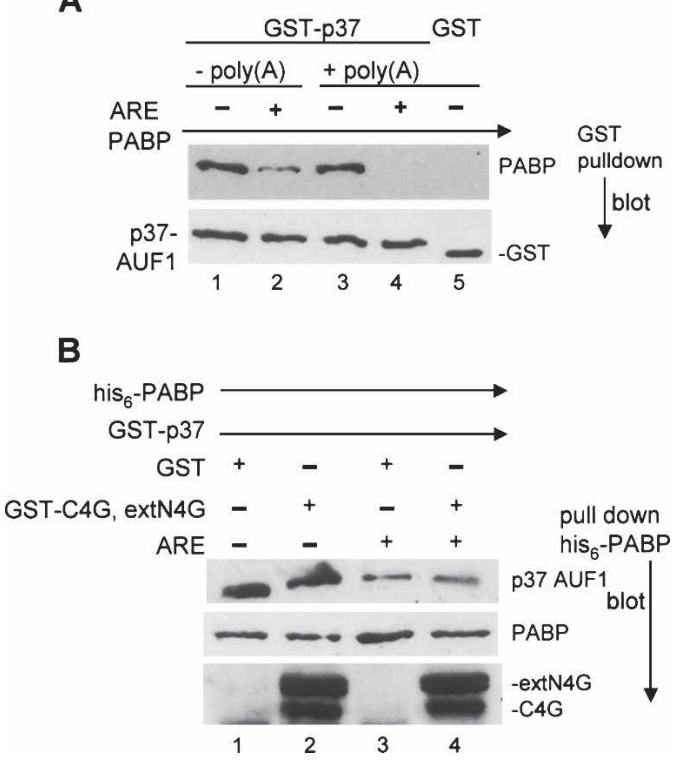

FIGURE 6. Simultaneous interaction of PABP with p37 AUF1 and components of the translation machinery. (A) Interaction of PABP with p37 AUF1 in the presence of poly $(A)$ RNA; $0.5 \mu \mathrm{g}$ of his $s_{6}$-PABP protein, $0.25 \mu \mathrm{M}$ poly $(A)$ RNA homopolymer, and $2 \mu \mathrm{M}$ ARE oligonucleotide were incubated as shown. GST-proteins were recovered by GSH-glutathione beads. The bound PABP and GST-AUF1 proteins were detected by immunoblot analysis. (B) Interaction of $\mathrm{p} 37$ AUF1 with PABP during simultaneous binding to eIF4G; $0.5 \mu \mathrm{g}$ of his $_{6}$-PABP and GST-p37 proteins was incubated with $0.5 \mu \mathrm{g}$ of GSTextN4G or GST-C4G proteins (or replaced with GST protein for control), in the presence or the absence of $1 \mu \mathrm{M}$ ARE RNA oligonucleotide in two different reactions. After $30 \mathrm{~min}$ of incubation, the two reactions were mixed for an additional $1 \mathrm{~h}$, followed by addition of TALON resin to recover his ${ }_{6}-\mathrm{PABP}$ protein. After washing, the bound p37 AUF1, PABP, and GST-eIF4G proteins were detected by immunoblot analysis. Typical results are shown. results indicate that monomeric or oligomerized PABP on poly(A) tails can interact directly with AUF1, but only if AUF1 is no longer bound to the ARE.

Many previous studies support the notion that the rapid turnover of ARE-mRNA often requires ongoing translation of the mRNA (Savant-Bhonsale and Cleveland 1992; Aharon and Schneider 1993; Curatola et al. 1995; Winstall et al. 1995). When active translation was blocked by cyclohexamide or by a strong hairpin structure, ARE-mRNAs were stabilized and resistant to rapid decay (Rajagopalan and Malter 1996). Since both PABP and AUF1 can interact with eIF4G, we next investigated whether the interaction of p37 AUF1 with PABP is affected by their mutual binding to eIF4G. PABP and p37 AUF1 were incubated with their eIF4G interaction partners in separate fractions for $30 \mathrm{~min}$ ( His $_{6}$-PABP with GST-extN4G; GST-p37 with GST-C4G). Fractions were then mixed to allow the interaction of p37 AUF1 with PABP. After a 60-min incubation, the p37 AUF1 bound to PABP was recovered by association with $\mathrm{His}_{6}$-PABP protein using TALON resin. Addition of ARE RNA reduced the binding of $\mathrm{p} 37$ AUF1 to $\mathrm{His}_{6}$-PABP by approximately threefold (Fig. 6B). Addition of the C4G and extN4G fragments, however, did not significantly influence the interaction between p37 AUF1 and $\mathrm{His}_{6}$-PABP, regardless of the presence or the absence of the ARE RNA (Fig. $6 \mathrm{~B})$. These results therefore suggest that PABP and p37 AUF1 can bind simultaneously to different regions of eIF4G, without a major alteration of the PABP-p37 AUF1 interaction.

\section{Interaction of PABP with elF4G is by enhanced by $\operatorname{poly}(\mathrm{A})$ RNA but not altered by the $\mathrm{p} 37$ AUF1-ARE complex}

It was previously demonstrated that interaction of $\mathrm{PABP}$ with eIF4G, potentially by circularizing the actively translated mRNA transcript (Wells et al. 1998), may play a vital role in maintaining the stability and translatability of the mRNA (Tarun et al. 1997; Imataka et al. 1998). The disruption of such a "closed loop" interaction could potentially destabilize mRNAs and, if carried out selectively, might lead to the rapid degradation of ARE-mRNAs. To address whether $\mathrm{p} 37$ AUF1 promotes the rapid decay of ARE-mRNAs by interfering with the PABP-eIF4G interaction, we investigated the effect of p37 AUF1 and ARE RNA on the association of PABP with eIF4G. Purified GSTextN4G recombinant protein was incubated with $\mathrm{His}_{6-}$ PABP protein in the presence or the absence of p37 AUF1 protein, with or without the addition of ARE RNA, as indicated. GST-eIF4G fusion protein was recovered by GST pull-down, and the bound $\mathrm{PABP}$ protein was resolved by SDS-PAGE and identified by immunoblot analysis. Addition of the ARE RNA caused a slight decrease $(\sim 50 \%)$ in the amount of PABP associated with GST-extN4G (Fig. 7, cf. lanes 1 and 2), possibly due to weak PABP interaction 


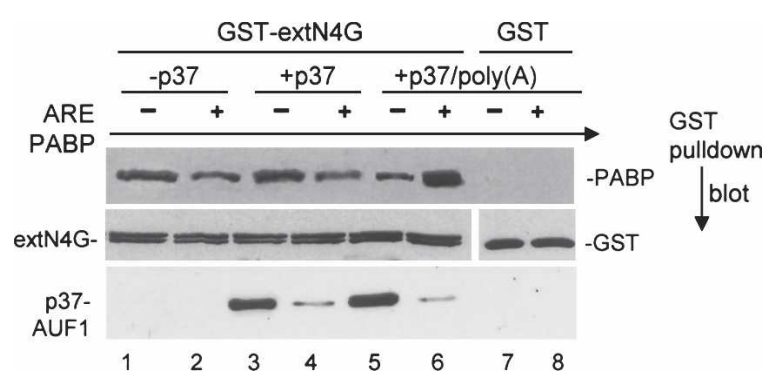

FIGURE 7. Effect of poly $(A)$ and the ARE on interaction of PABP and AUF1 with eIF4G. Effect of p37 AUF1, poly $(A)$, and ARE RNA oligonucleotide on the interaction of PABP with eIF4G. One microgram $(1 \mu \mathrm{g})$ of GST-extN4G, $0.5 \mu \mathrm{g}$ of his6-PABP protein, $0.5 \mu \mathrm{M}$ p37 AUF1 protein, and $2.5 \mu \mathrm{M}$ ARE RNA oligonucleotide were added as indicated. GST-proteins were recovered by glutathione beads. Proteins were resolved by SDS-PAGE and detected by immunoblot analysis.

with the ARE. In addition, the presence of p37 AUF1 did not alter the amount of PABP bound to eIF4G (Fig. 7, cf. lanes 2-4), regardless of its association with the ARE RNA (Fig. 7, lanes 3,4). Therefore, AUF1 does not disrupt PABPeIF4G interaction. Importantly, the binding of AUF1 to the ARE RNA actually disrupted its binding to PABP. Thus, displacement of p37 AUF1 from the ARE, possibly during ribosome transit, would be expected to promote its binding to PABP.

We therefore further examined the interactions of these components in a manner that more closely mimics the assembly of the translation machinery by addition of poly(A) to the interaction analysis (Fig. 7, lanes 5,6). Without the TNF $\alpha$ ARE RNA, addition of poly(A) RNA (with p37 AUF1) did not enhance binding between PABP and extN4G (Fig. 7, cf. lanes 1 and 5), whereas when combined, the ARE and poly(A) strongly increased (approximately fourfold) the amount of PABP bound to extN4G (Fig. 7, cf. lanes 1-5 and 6). These data are consistent with a prior report that indicated that binding of PABP to the poly(A) tail might strengthen the eIF4G-PABP interaction, thereby stabilizing mRNA (Coller et al. 1998). Since addition of the ARE RNA strongly increased the binding of PABP to extN4G, these data suggest that binding of p37 AUF1 to the ARE RNA blocks AUF1 interaction with PABP, thereby promoting eIF4G/PABP interaction, which may be important for mRNA stability. Conversely, in the absence of AUF1 binding to the ARE, AUF1 binds more strongly to PABP, which might also contribute to mRNA instability.

\section{DISCUSSION}

It has been shown that decay of ARE-mRNAs is closely associated with ongoing translation of the mRNA and that AUF1 promotes the rapid decay of ARE-mRNAs both in vivo and in vitro (Brewer 1991; Savant-Bhonsale and Cleveland 1992; Rajagopalan and Malter 1996; Sirenko et al. 1997). The mechanism by which AUF1 acts to promote
mRNA decay, however, remains unknown. AUF1 forms a complex in vivo with the translation apparatus and heat shock proteins (Laroia et al. 1999), but the direct interaction of AUF1 with other proteins that function in translation and mRNA decay has not been determined. There is also little understanding of the effect of AUF1ARE binding on these interactions. Using an in vitro interaction assay and synthetic ARE and poly(A) RNA, we have demonstrated that AUF1 isoforms strongly interact with the $\mathrm{C}$ terminus of eIF4G, independently of its binding to the ARE. AUF1 was also found to interact strongly and directly with PABP, which was opposed by heat shock protein Hsp70 and the binding of AUF1 to the ARE. AUF1 was also found to bind PABP while both proteins were associated with eIF4G.

We suggest a working model in which the concerted interactions between AUF1, the ARE, and specific components of the translation initiation apparatus regulate the rapid decay of ARE-mRNAs. The high-affinity association of p37 AUF1 with the C-terminal region of eIF4G could act to load p37 AUF1 onto the ARE in a cap-initiation complex and ribosome-dependent fashion, and/or promote AUF1 interaction with PABP. Importantly, our results reveal that p37 AUF1 has significantly reduced ability to directly interact with PABP while it is also bound to the ARE (Fig. 5). Since we have shown that AUF1 interacts in vivo with a complex that contains PABP and, as in this study, shown it does so directly, these results suggest that upon active translation of the ARE-mRNA, movement of the ribosome along the mRNA might displace AUF1 from the ARE (directly or indirectly). While it is still not known whether ribosomes transit into the $3^{\prime}$ noncoding region following termination of translation or disrupt $3^{\prime}$ RNA conformation thereby displacing proteins, precedent for this possibility can be found in the Y14 shuttling RNAbinding protein. Y14 protein is found in the exon junction complex on translationally inactive mRNAs and is removed with mRNA translation (Dostie and Dreyfuss 2002). Although the ARE is located $3^{\prime}$ to the termination codon, it needs to be noted that the fate of scanning ribosomes, the manner of their transition/recycling on mRNA, and translation-mediated alterations to mRNP structure at both the $5^{\prime}$ - and $3^{\prime}$-ends all remain to be determined. Thus, only in the absence of binding to the ARE can the p37 AUF1 protein bind PABP with high affinity.

The rapid degradation of p37 AUF1, and to some extent p40 AUF1, which is mediated by ubiquitination and the proteasome pathway (Laroia et al. 1999), could in principle lead to the codegradation of interacting PABP via its association as a complex. This, in turn, would then make the poly(A) tail more susceptible to deadenylation and result in degradation of the ARE-containing transcript. Nevertheless, we could find no evidence in vivo to support this possibility. We asked whether the interaction of AUF1 with PABP decreases PABP stability. Cells were treated with 
cycloheximide to block protein synthesis and assess the stabilities of free AUF1 and PABP and AUF1-PABP complexes in vivo, by conducting a pulse-chase analysis. We could find no difference in PABP stability in both free and AUF1 complexed forms (data not shown), suggesting that AUF1 does not function by causing the rapid codegradation of associated PABP. Instead, our data suggest that a block to ongoing translation would make PABP inaccessible to p37 AUF1 interaction because the binding of AUF1 to the AU-rich element largely diminishes this interaction. These biochemical data suggest that the link between ARE-mRNA decay and active mRNA translation might entail a mechanism driven by ribosomes that promotes AUF1 binding to PABP through AUF1 displacement from the ARE. Our data also suggest a mechanism for AUF1 function, whereby AUF1 interaction with PABP would remove it from the poly $(\mathrm{A})$ tail, not by codegradation but by stable complex formation separate from the cap-initiation complex. Consistent with our previous report that induction of Hsp70 stabilizes ARE-mRNAs (Laroia et al. 1999), Hsp70 disrupted the interaction between p37 AUF1 and PABP in vitro, possibly through the direct association and sequestration of p37 AUF1 by Hsp70.

The exosome has been implicated in ARE-mRNA degradation in mammalian cellular extracts (Chen et al. 2001; Mukherjee et al. 2002). In one study, the exosome appeared to be mainly responsible for degradation of the mRNA body but not the poly(A) tail (Chen et al. 2001), while another study suggested a correlation between exosome activity and mRNA deadenylation, since depletion of the exosome strongly inhibited deadenylation (Mukherjee et al. 2002). Some ARE-binding proteins (including AUF1, TTP, and KSRP) were found associated with the exosome. Whether the exosome directly recognizes the ARE or is recruited to the ARE via ARE-binding proteins remains an unresolved question. It is possible that AUF1 also participates in the decay of the mRNA body via its interaction with the exosome.

An alternate mechanism by which AUF1 might promote ARE-mRNA decay is that the interaction of AUF1 with eIF4G may regulate deadenylation by affecting the capbinding complex and the accessibility of the cap structure to PARN, the major poly (A)-specific deadenylase. PARN specifically and directly interacts with the $5^{\prime}$-cap structure, and cap-PARN interactions play an important role in determining the rate of deadenylation (Gao et al. 2000). The addition of purified eIF4E protein to the in vitro decay system, which competes with PARN for the cap structure, efficiently inhibits the deadenylation of capped RNA. Although the ARE itself was not required for the deadenylase to cross-link to the cap structure, AUF1 may indirectly control cap accessibility via its direct interaction with eIF4G and alter the composition of cap-binding complexes upon the translation of mRNA. The interaction of the PARN-cap structure is greatly stimulated by a poly(A) tail (Gao et al. 2000), which raises the possibility that the initial exposure of a portion of the poly(A) tail resulting from AUF1-PABP interaction could be responsible for loading PARN onto the cap structure. Alternatively, AUF1 could directly facilitate efficient deadenylation by displacing PABP from poly(A) as described above. Finally, it cannot be excluded that AUF1, together with other translation factors and/or AU-binding proteins, may also contribute to ARE-stimulated decapping of the mRNA transcript (Gao et al. 2001).

In summary, results from this study support the hypothesis that the direct interaction of AUF1 with eIF4G and PABP, which is impaired by ARE-binding and heat shock proteins, links the rapid degradation of ARE-mRNAs with the translational machinery and couples ARE-mRNA stabilization with induction of Hsp70 expression upon heat shock stress.

\section{MATERIALS AND METHODS}

\section{Expression vectors and cloning}

Bacterial expression plasmids were constructed in plasmid pGEX4T-1 (Pharmacia), which encodes full-length fusions of cDNAs corresponding to the different AUF1 isoforms and an N-terminal GST peptide. Briefly, cloning involved polymerase chain reaction (PCR) amplification of the coding regions of fulllength p37, p40, p42, and p45 AUF1 isoforms, as well as p37 AUF1 truncation mutants (p37 $\Delta$ N78, amino acids 79-287; p37 $\Delta$ C45, amino acids $1-242 ; \mathrm{p} 37 \Delta \mathrm{N}$, amino acids $79-242$ ). PCR products were purified by gel-electrophoresis, digested with BamHI and XhoI, and then cloned into pGEX-4TI (Amersham-Pharmacia) digested with the same enzymes. To generate $\mathrm{His}_{6}$-tagged AUF1 proteins, cDNAs were PCR-cloned into pET-23b (Novagen) using the BamHI and XhoI sites, allowing in-frame fusion with the $\mathrm{N}$-terminal T7 tag and the C-terminal histidine ( $\left.\mathrm{His}_{6}\right)$ tag. Specific details of cloning procedures are available upon request. Correct cloning was confirmed by DNA sequencing analysis. To construct Flag-tagged (N-terminal) AUF1 expression vectors, the coding region of AUF1 cDNAs flanked by HindIII and EcoRI sites was PCR-synthesized and cloned into pFLAG-CMV2 (Sigma) at these sites. Plasmids expressing glutathione S-transferase (GST) fusions of N-terminal, middle, and C-terminal segments of eIF4GI have been described previously in Cuesta et al. (2000a). A plasmid expressing extended (ext) N-terminal eIF4GI (amino acids 1-401) was constructed into the vector pGEXKG (Pharmacia) for expression of the GST-extN4G fusion protein. pGEX2T-PABP was provided by Dr. J. Bag (University of Guelph). Plasmid pET28a-PABP was kindly provided by Dr. M. Kiledjian (Rutgers University).

\section{Recombinant proteins and antibodies}

All recombinant proteins were expressed in BL21(DE3) E. coli cells. To purify GST-tagged proteins (AUF1 isoforms, p37 AUF1 mutants, eIF4G fragments, PABP), bacterial cultures were induced for $2 \mathrm{~h}$ in LB containing $1 \mathrm{mM}$ IPTG. Cells were resuspended in 
lysis buffer $(10 \mathrm{mM}$ Tris- $\mathrm{HCl}$ at $\mathrm{pH} 8.0,100 \mathrm{mM} \mathrm{NaCl}, 1 \mathrm{mM}$ EDTA, $0.7 \mathrm{mg} / \mathrm{mL}$ lysozyme, 1\% NP-40, 1 mM DTT, 1 mM PMSF) and sonicated for $2 \mathrm{~min}$. After addition of $\mathrm{NaCl}$ (final $450 \mathrm{mM}$ ), cell debris was removed by centrifugation at 15,000 rpm for $30 \mathrm{~min}$ at $4^{\circ} \mathrm{C}$. The cleared lysate was incubated with glutathioneSepharose $4 \mathrm{~B}$ (Pharmacia) at $4^{\circ} \mathrm{C}$ for $2 \mathrm{~h}$. The column was washed with $30 \mathrm{~mL}$ of buffer $\mathrm{A}\left(20 \mathrm{mM} \mathrm{NaH}_{2} \mathrm{PO}_{4}\right.$ at $\mathrm{pH} 7.3,450 \mathrm{mM} \mathrm{NaCl}$, $0.5 \mathrm{mM}$ EDTA, $1 \%$ Triton X-100, $1 \mathrm{mM}$ DTT, $1 \mathrm{mM}$ PMSF) followed by $20 \mathrm{~mL}$ of buffer B $\left(150 \mathrm{mM} \mathrm{NaCl}, 20 \mathrm{mM} \mathrm{NaH}_{2} \mathrm{PO}_{4}\right.$ at $\mathrm{pH} 7.3,0.5 \mathrm{mM}$ EDTA, $1 \mathrm{mM}$ DTT, $1 \mathrm{mM}$ PMSF). GST proteins were eluted with elution buffer $(50 \mathrm{mM}$ Tris- $\mathrm{HCl}$ at $\mathrm{pH}$ 8.0, $10 \mathrm{mM}$ glutathione, $1 \mathrm{mM}$ PMSF, $1 \mathrm{mM}$ DTT). The GST tag was removed from GST-p37 by thrombin cleavage (Pharmacia).

His-tagged forms of proteins (AUF1, PABP) were purified similarly as described above except that cells were lysed in a $\mathrm{His}_{6}$-extract buffer $\left(50 \mathrm{mM} \mathrm{NaH} \mathrm{PO}_{4}\right.$ at $\mathrm{pH} 7.0,300 \mathrm{mM}$ $\mathrm{NaCl}, 0.5 \% \mathrm{NP}-40,0.7 \mathrm{mg} / \mathrm{mL}$ lysozyme, $1 \mathrm{mM}$ PMSF). Some preparations of PABP were treated with a ribonuclease cocktail to eliminate contaminating RNA (Ambion), as indicated. The cleared lysate was then mixed with TALON metal affinity resin (Clontech). After washing with $\mathrm{His}_{6}$-extract buffer, bound proteins were eluted with elution buffer $\left(50 \mathrm{mM} \mathrm{NaH}_{2} \mathrm{PO}_{4}\right.$ at $\mathrm{pH} 8.0$, $300 \mathrm{mM} \mathrm{NaCl}, 100 \mathrm{mM}$ imidazole). His $_{6}$-tagged p37 AUF1 was also expressed in $\mathrm{Hi} 5$ insect cells and purified by TALON chromatography. All purified proteins were dialyzed against PBS supplemented with $10 \%$ glycerol and stored at $-80^{\circ} \mathrm{C}$. Purified proteins were judged to be $>95 \%$ pure and intact by SDSpolyacrylamide gel electrophoresis (SDS-PAGE) and Coomassie blue staining of proteins. Protein concentrations were determined using BioRad Reagent as described by the manufacturer. Recombinant Hsp70 and Hsc70 proteins were purchased from Stressgen (Canada). Anti-Flag monoclonal antibody M2 was purchased from Sigma. Antibody to Hsp70 (w27, sc-24) and anti-GST antibodies were from Santa Cruz, Inc. Anti-T7 and anti$\mathrm{His}_{6}$ monoclonal antibodies were from Novagen and Roche, respectively. Anti-AUF1 polyclonal antibody and anti-PABP (10E10) monoclonal antibodies were kindly provided by Drs. Gary Brewer (University of Medicine and Dentistry of New Jersey) and Gideon Dreyfuss (University of Pennsylvania), respectively.

\section{RNA substrate}

Studies used an RNA oligonucleotide corresponding to TNF $\alpha$ 3'-UTR AU-rich sequence 5'-UGAUUAUUUAUUAUUUAUUU AUUAUUUAUUUAUUUAG-3', which was synthesized by Dharmacon Research, Inc. After deprotection, the RNA oligonucleotide was reconstituted with sterile water to $50 \mu \mathrm{M}$ and stored at $-20^{\circ} \mathrm{C}$. Poly(A) RNA homopolymers (average length 500 ribonucleotides) were purchased from Pharmacia Biotech.

\section{In vitro AUF1 protein interaction analysis}

Recombinant proteins were incubated in $100 \mu \mathrm{L}$ of Binding Buffer (10 mM HEPES-KOH at $\mathrm{pH} 7.5,100 \mathrm{mM}$ potassium acetate at $\mathrm{pH}$ 7.5, $5 \mathrm{mM}$ magnesium acetate, $0.5 \% \mathrm{NP}-40,0.1 \mathrm{mg} / \mathrm{mL} \mathrm{BSA}$ ) for $1 \mathrm{~h}$ at $4^{\circ} \mathrm{C}$ with the presence or absence of $1 \mu \mathrm{M}$ TNF ARE RNA oligonucleotide. Then $15 \mu \mathrm{L}$ of glutathione-Sepharose $4 \mathrm{~B}$ slurry was added, followed by incubation for $1 \mathrm{~h}$ at $4^{\circ} \mathrm{C}$. Bound proteins were washed three times with Binding Buffer, eluted by boiling in SDS-loading dye, then resolved by $10 \%$ SDS-PAGE.
Immunoblotting was performed using the antibodies indicated. Protein-protein cross-linking was carried out in a $20-\mu \mathrm{L}$ reaction containing $10 \mathrm{mM}$ HEPES-KOH ( $\mathrm{pH} 7.4$ ), $100 \mathrm{mM}$ magnesium acetate, $5 \mathrm{mM}$ magnesium acetate. Purified recombinant p37 AUF1 protein $(1 \mu \mathrm{M})$ was incubated in the presence or the absence of TNF $\alpha$ ARE RNA oligonucleotide at room temperature for $15 \mathrm{~min}$ followed by addition of DSP (dithio-bis(succinimidyl propionate); Pierce). Reactions were incubated for an additional 45 min. Reaction products were resolved by SDS-9\% PAGE gel without reducing agents. Immunoblot analyses were performed with polyclonal antibodies to AUF1 and the SuperSignal Chemiluminescent Detection Kit (Pierce).

\section{PABP half-life analysis}

$\mathrm{CHO}$ cells were treated with cycloheximide for up to $18 \mathrm{~h}$ at $100 \mu \mathrm{g} / \mathrm{mL}$, and extracts were produced in NP-40 lysis buffer (described above) and cleared by microcentrifugation at $10,000 \mathrm{~g}$ for $3 \mathrm{~min}$ at $4^{\circ} \mathrm{C}$. Equal amounts of lysate were used for immunoblot analysis or subjected to immunoprecipitation with anti-AUF1 antibodies, followed by immunoblot analysis of AUF1 and PABP complexes. Autoradiograms were quantified by densitometry.

\section{ACKNOWLEDGMENTS}

We thank Gideon Dreyfuss for antibodies to AUF1 and PABP, Gary Brewer for antibodies to AUF1, M. Kiledjian and J. Bag for plasmids, and G. Wilson for helpful advice on cross-linking studies. We also thank members of the lab for critical review of the manuscript. This work was supported by NIH grant GM60428 to R.J.S.

Received November 30, 2005; accepted February 7, 2006.

\section{REFERENCES}

Aharon, T. and Schneider, R.J. 1993. Selective destabilization of shortlived mRNAs with the granulocyte-macrophage colony-stimulating factor AU-rich $3^{\prime}$ noncoding region is mediated by a cotranslational mechanism. Mol. Cell. Biol. 13: 1971-1980.

Bradley, C.A., Padovan, J.C., Thompson, T.L., Benoit, C.A., Chait, B.T., and Rhoads, R.E. 2002. Mass spectrometric analysis of the $\mathrm{N}$ terminus of translational initiation factor eIF4G-1 reveals novel isoforms. J. Biol. Chem. 277: 12559-12571.

Brewer, G. 1991. An A + U-rich element RNA-binding factor regulates c-myc mRNA stability in vitro. Mol. Cell. Biol. 11: 2460-2466.

Brewer, G. and Ross, J. 1988. Poly(A) shortening and degradation of the 3' A + U-rich sequences of human c-myc mRNA in a cell-free system. Mol. Cell. Biol. 8: 1697-1708.

Brys, A. and Maizels, N. 1994. LR1 regulates c-myc transcription in B-cell lymphomas. Proc. Natl. Acad. Sci. 91: 4915-4919.

Bukau, B. and Horwich, A.L. 1998. The Hsp70 and Hsp60 chaperone machines. Cell 92: 351-366.

Byrd, M.P., Zamora, M., and Lloyd, R.E. 2002. Generation of multiple isoforms of eukaryotic translation initiation factor 4GI by use of alternate translation initiation codons. Mol. Cell. Biol. 22: 44994511.

Carballo, E., Lai, W.S., and Blackshear, P.J. 2000. Evidence that tristetraprolin is a physiological regulator of granulocyte-macrophage colony-stimulating factor messenger RNA deadenylation and stability. Blood 95: 1891-1899.

Chen, C.Y., Gherzi, R., Ong, S.E., Chan, E.L., Raijmakers, R., Pruijn, G.J., Stoecklin, G., Moroni, C., Mann, M., and Karin, M. 
2001. AU binding proteins recruit the exosome to degrade AREcontaining mRNAs. Cell 107: 451-464.

Coller, J.M., Gray, N.K., and Wickens, M.P. 1998. mRNA stabilization by poly(A) binding protein is independent of poly(A) and requires translation. Genes \& Dev. 12: 3226-3235.

Cuesta, R., Laroia, G., and Schneider, R.J. 2000a. Chaperone hsp27 inhibits translation during heat shock by binding eIF4G and facilitating dissociation of cap-initiation complexes. Genes \& Dev. 14: $1460-1470$.

Cuesta, R., Xi, Q., and Schneider, R.J. 2000b. Adenovirus-specific translation by displacement of kinase Mnk1 from cap-initiation complex eIF4F. EMBO J. 19: 3465-3474.

Curatola, A.M., Nadal, M.S., and Schneider, R.J. 1995. Rapid degradation of AU-rich element (ARE) mRNAs is activated by ribosome transit and blocked by secondary structure at any position 5' to the ARE. Mol. Cell. Biol. 15: 6331-6340.

DeMaria, C.T., Sun, Y., Long, L., Wagner, B.J., and Brewer, G. 1997. Structural determinants in AUF1 required for high affinity binding to A + U-rich elements. J. Biol. Chem. 272: 27635-27643.

Dostie, J. and Dreyfuss, G. 2002. Translation is required to remove Y14 from mRNAs in the cytoplasm. Curr. Biol. 12: 1060-1067.

Eversole, A. and Maizels, N. 2000. In vitro properties of the conserved mammalian protein hnRNP D suggest a role in telomere maintenance. Mol. Cell. Biol. 20: 5425-5432.

Fan, X.C. and Steitz, J.A. 1998. Overexpression of HuR, a nuclearcytoplasmic shuttling protein, increases the in vivo stability of ARE-containing mRNAs. EMBO J. 17: 3448-3460.

Ford, L.P. and Wilusz, J. 1999. An in vitro system using HeLa cytoplasmic extracts that reproduces regulated mRNA stability. Methods 17: 21-27.

Fuentes-Panana, E.M., Peng, R., Brewer, G., Tan, J., and Ling, P.D. 2000. Regulation of the Epstein-Barr virus C promoter by AUF1 and the cyclic AMP/protein kinase A signaling pathway. J. Virol. 74: $8166-8175$.

Gao, M., Fritz, D.T., Ford, L.P., and Wilusz, J. 2000. Interaction between a poly(A)-specific ribonuclease and the $5^{\prime}$ cap influences mRNA deadenylation rates in vitro. Mol. Cell 5: 479-488.

Gao, M., Wilusz, C.J., Peltz, S.W., and Wilusz, J. 2001. A novel mRNA-decapping activity in HeLa cytoplasmic extracts is regulated by AU-rich elements. EMBO J. 20: 1134-1143.

Guhaniyogi, J. and Brewer, G. 2001. Regulation of mRNA stability in mammalian cells. Gene 265: 11-23.

Hanakahi, L.A., Dempsey, L.A., Li, M.J., and Maizels, N. 1997. Nucleolin is one component of the B cell-specific transcription factor and switch region binding protein, LR1. Proc. Natl. Acad. Sci. 94: 3605-3610.

Imataka, H., Gradi, A., and Sonenberg, N. 1998. A newly identified $\mathrm{N}$-terminal amino acid sequence of human eIF4G binds poly(A)binding protein and functions in poly(A)-dependent translation. EMBO J. 17: 7480-7489.

Kajita, Y., Nakayama, J., Aizawa, M., and Ishikawa, F. 1995. The UUAG-specific RNA binding protein, heterogeneous nuclear ribonucleoprotein D0. Common modular structure and binding properties of the 2xRBD-Gly family. J. Biol. Chem. 270: 22167-22175.

Kiledjian, M., DeMaria, C.T., Brewer, G., and Novick, K. 1997. Identification of AUF1 (heterogeneous nuclear ribonucleoprotein D) as a component of the $\alpha$-globin mRNA stability complex. Mol. Cell. Biol. 17: 4870-4876.

Lai, W.S., Carballo, E., Thorn, J.M., Kennington, E.A., and Blackshear, P.J. 2000. Interactions of $\mathrm{CCCH}$ zinc finger proteins with mRNA. Binding of tristetraprolin-related zinc finger proteins to Au-rich elements and destabilization of mRNA. J. Biol. Chem. 275: $17827-17837$.

Laroia, G. and Schneider, R.J. 2002. Alternate exon insertion controls selective ubiquitination and degradation of different AUF1 protein isoforms. Nucleic Acids Res. 30: 3052-3058.

Laroia, G., Cuesta, R., Brewer, G., and Schneider, R.J. 1999. Control of mRNA decay by heat shock-ubiquitin-proteasome pathway. Science 284: 499-502.
Laroia, G., Sarkar, B., and Schneider, R.J. 2002. Ubiquitin-dependent mechanism regulates rapid turnover of AU-rich cytokine mRNAs. Proc. Natl. Acad. Sci. 99: 1842-1846.

Levy, N.S., Chung, S., Furneaux, H., and Levy, A.P. 1998. Hypoxic stabilization of vascular endothelial growth factor mRNA by the RNA-binding protein HuR. J. Biol. Chem. 273: 6417-6423.

Loflin, P., Chen, C.Y., and Shyu, A.B. 1999. Unraveling a cytoplasmic role for hnRNP D in the in vivo mRNA destabilization directed by the AU-rich element. Genes \& Dev. 13: 1884-1897.

Ma, W.J., Cheng, S., Campbell, C., Wright, A., and Furneaux, H. 1996. Cloning and characterization of HuR, a ubiquitously expressed Elav-like protein. J. Biol. Chem. 271: 8144-8151.

Mayeda, A. and Krainer, A.R. 1992. Regulation of alternative premRNA splicing by hnRNP A1 and splicing factor SF2. Cell 68: 365-375.

Mukherjee, D., Gao, M., O’Connor, J.P., Raijmakers, R., Pruijn, G., Lutz, C.S., and Wilusz, J. 2002. The mammalian exosome mediates the efficient degradation of mRNAs that contain AU-rich elements. EMBO J. 21: 165-174.

Peng, S.S., Chen, C.Y., Xu, N., and Shyu, A.B. 1998. RNA stabilization by the AU-rich element binding protein, HuR, an ELAV protein. EMBO J. 17: 3461-3470.

Petit, F., Jarrousse, A.S., Dahlmann, B., Sobek, A., Hendil, K.B., Buri, J., Briand, Y., and Schmid, H.P. 1997. Involvement of proteasomal subunits $\zeta$ and $\tau$ in RNA degradation. Biochem. J. 326: 93-98.

Pinol-Roma, S. and Dreyfuss, G. 1992. Shuttling of pre-mRNA binding proteins between nucleus and cytoplasm. Nature 355: 730-732.

Pinol-Roma, S., Choi, Y.D., Matunis, M.J., and Dreyfuss, G. 1988. Immunopurification of heterogeneous nuclear ribonucleoprotein particles reveals an assortment of RNA-binding proteins. Genes \& Dev. 2: 215-227.

Pioli, P.A., Hamilton, B.J., Connolly, J.E., Brewer, G., and Rigby, W.F. 2002. Lactate dehydrogenase is an AU-rich element-binding protein that directly interacts with AUF1. J. Biol. Chem. 277: 3573835745.

Pouch, M.N., Petit, F., Buri, J., Briand, Y., and Schmid, H.P. 1995. Identification and initial characterization of a specific proteasome (prosome) associated RNase activity. J. Biol. Chem. 270: 2202322028.

Rajagopalan, L.E. and Malter, J.S. 1996. Turnover and translation of in vitro synthesized messenger RNAs in transfected, normal cells. J. Biol. Chem. 271: 19871-19876.

Rodriguez-Pascual, F., Hausding, M., Ihrig-Biedert, I., Furneaux, H., Levy, A.P., Forstermann, U., and Kleinert, H. 2000. Complex contribution of the $3^{\prime}$-untranslated region to the expressional regulation of the human inducible nitric-oxide synthase gene. J. Biol. Chem. 275: 26040-26049.

Sarkar, B., Lu, J.Y., and Schneider, R.J. 2003a. Nuclear import and export functions in the different isoforms of the AUF1/heterogeneous nuclear ribonucleoprotein protein family. J. Biol. Chem. 278: 20700-20707.

Sarkar, B., Xi, Q., He, C., and Schneider, R.J. 2003b. Selective degradation of AU-rich mRNAs promoted by the p37 AUF1 protein isoform. Mol. Cell. Biol. 23: 6685-6693.

Savant-Bhonsale, S. and Cleveland, D.W. 1992. Evidence for instability of mRNAs containing AUUUA motifs mediated through translation-dependent assembly of a $>20$ S degradation complex. Genes \& Dev. 6: 1927-1939.

Schiavi, S.C., Wellington, C.L., Shyu, A.B., Chen, C.Y., Greenberg, M.E., and Belasco, J.G. 1994. Multiple elements in the $\mathrm{c}$-fos protein-coding region facilitate mRNA deadenylation and decay by a mechanism coupled to translation. J. Biol. Chem. 269: $3441-3448$

Schmid, H.P., Pouch, M.N., Petit, F., Dadet, M.H., Badaoui, S., Boissonnet, G., Buri, J., Norris, V., and Briand, Y. 1995. Relationships between proteasomes and RNA. Mol. Biol. Rep. 21: 43-47.

Shaw, G. and Kamen, R. 1986. A conserved AU sequence from the $3^{\prime}$ untranslated region of GM-CSF mRNA mediates selective mRNA degradation. Cell 46: 659-667. 
Shyu, A.B., Greenberg, M.E., and Belasco, J.G. 1989. The c-fos transcript is targeted for rapid decay by two distinct mRNA degradation pathways. Genes \& Dev. 3: 60-72.

Shyu, A.B., Belasco, J.G., and Greenberg, M.E. 1991. Two distinct destabilizing elements in the c-fos message trigger deadenylation as a first step in rapid mRNA decay. Genes \& Dev. 5: 221-231.

Sirenko, O.I., Lofquist, A.K., DeMaria, C.T., Morris, J.S., Brewer, G., and Haskill, J.S. 1997. Adhesion-dependent regulation of an A + U-rich element-binding activity associated with AUF1. Mol. Cell. Biol. 17: 3898-3906.

Stoecklin, G., Ming, X.F., Looser, R., and Moroni, C. 2000. Somatic mRNA turnover mutants implicate tristetraprolin in the interleukin-3 mRNA degradation pathway. Mol. Cell. Biol. 20: 3753-3763.

Tarun Jr., S.Z., Wells, S.E., Deardorff, J.A., and Sachs, A.B. 1997. Translation initiation factor eIF4G mediates in vitro poly(A) taildependent translation. Proc. Natl. Acad. Sci. 94: 9046-9051.

Taylor, G.A., Carballo, E., Lee, D.M., Lai, W.S., Thompson, M.J., Patel, D.D., Schenkman, D.I., Gilkeson, G.S., Broxmeyer, H.E., Haynes, B.F., et al. 1996. A pathogenetic role for TNF $\alpha$ in the syndrome of cachexia, arthritis, and autoimmunity resulting from tristetraprolin (TTP) deficiency. Immunity 4: 445-454.

Wagner, B.J., DeMaria, C.T., Sun, Y., Wilson, G.M., and Brewer, G. 1998. Structure and genomic organization of the human AUF1 gene: Alternative pre-mRNA splicing generates four protein isoforms. Genomics 48: 195-202.
Wang, W., Caldwell, M.C., Lin, S., Furneaux, H., and Gorospe, M. 2000. HuR regulates cyclin A and cyclin B1 mRNA stability during cell proliferation. EMBO J. 19: 2340-2350.

Wells, S.E., Hillner, P.E., Vale, R.D., and Sachs, A.B. 1998. Circularization of mRNA by eukaryotic translation initiation factors. Mol. Cell 2: 135-140.

Wilson, T. and Treisman, R. 1988. Removal of poly(A) and consequent degradation of c-fos mRNA facilitated by $3^{\prime}$ AU-rich sequences. Nature 336: 396-399.

Wilson, G.M., Sun, Y., Lu, H., and Brewer, G. 1999. Assembly of AUF1 oligomers on U-rich RNA targets by sequential dimer association. J. Biol. Chem. 274: 33374-33381.

Wilson, G.M., Sutphen, K., Chuang, K., and Brewer, G. 2001. Folding of A + U-rich RNA elements modulates AUF1 binding. Potential roles in regulation of mRNA turnover. J. Biol. Chem. 276: 86958704.

Winstall, E., Gamache, M., and Raymond, V. 1995. Rapid mRNA degradation mediated by the c-fos 3' AU-rich element and that mediated by the granulocyte-macrophage colony-stimulating factor 3' AU-rich element occur through similar polysome-associated mechanisms. Mol. Cell. Biol. 15: 3796-3804.

$\mathrm{Xu}$, N., Chen, C.Y., and Shyu, A.B. 1997. Modulation of the fate of cytoplasmic mRNA by AU-rich elements: Key sequence features controlling mRNA deadenylation and decay. Mol. Cell. Biol. 17: 4611-4621. 

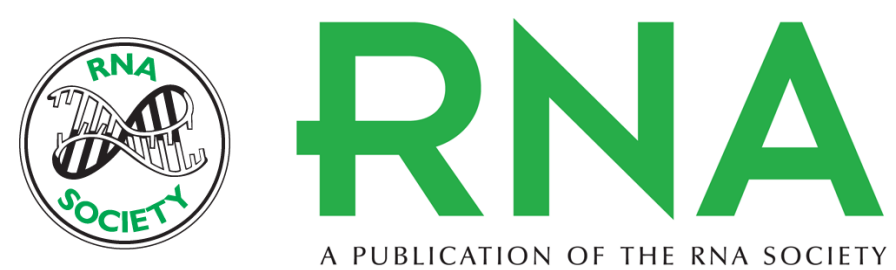

\section{Assembly of AUF1 with elF4G-poly(A) binding protein complex suggests a translation function in AU-rich mRNA decay}

Jin-Yu Lu, Naomi Bergman, Navid Sadri, et al.

RNA 2006 12: 883-893

References This article cites 63 articles, 48 of which can be accessed free at:

http://rnajournal.cshlp.org/content/12/5/883.full.html\#ref-list-1

License

Email Alerting Receive free email alerts when new articles cite this article - sign up in the box at the Service top right corner of the article or click here. 